\title{
Editorial
}

\section{Theory and Applications of Fractional Order Systems 2016}

\author{
Riccardo Caponetto, ${ }^{1}$ Juan J. Trujillo, ${ }^{2}$ and Josè A. Tenereiro Machado ${ }^{3}$ \\ ${ }^{1}$ Department of Electrical, Electronics and Computer Engineering, University of Catania, Viale A. Doria 6, 95125 Catania, Italy \\ ${ }^{2}$ Department of Mathematical Analysis, University of La Laguna, C/Astr. Francisco Sanchez, s/n, La Laguna, 38271 Tenerife, Spain \\ ${ }^{3}$ Department of Electrical Engineering, ISEP-Institute of Engineering, Polytechnic of Porto, \\ Rua Dr. Antonio Bernardino de Almeida 431, 4200-072 Porto, Portugal
}

Correspondence should be addressed to Riccardo Caponetto; riccardo.caponetto@dieei.unict.it

Received 4 September 2016; Accepted 5 September 2016

Copyright ( 92016 Riccardo Caponetto et al. This is an open access article distributed under the Creative Commons Attribution License, which permits unrestricted use, distribution, and reproduction in any medium, provided the original work is properly cited.

In the last decades noninteger differentiation became a popular tool for modeling the complex behaviours of physical systems from diverse domains such as mechanics, electricity, chemistry, biology, and economics. Numerous studies have validated the novel perspective demonstrating fractional order models that better characterize many real-world physical systems by means of differential operators of noninteger order. The long-range temporal or spatial dependence phenomena inherent to the fractional order systems (FOS) present unique and intriguing peculiarities, not supported by their integer order counterpart, which raise exciting challenges and opportunities related to the development of modelling, control, and estimation methodologies involving fractional order dynamics.

The purpose of this special issue, following the 2014 one, is to continue drawing attention of the scientific community to some recent advances and possible applications of fractional order systems and to ensure the corresponding dissemination. The issue includes a collection of papers in the area of FOS and some leading and emerging specialists in the area present their latest results.

In the 2014 issue, 13 papers of 24 submitted have been accepted; in this issue 14 of 42 have been published, outlining, therefore, the increasing interest at the topic.

A short description of the addressed topics is as follows.

In "Convergence of Laguerre Impulse Response Approximation for Noninteger Order Systems" new results on the Laguerre Impulse Response Approximation method of noninteger order systems are presented. Among others, a new proof of $L_{1}$ convergence of approximation is given, allowing less strict assumptions. Additionally more general results are given including one regarding functions that are in the joint part of $L_{1}$ and $L_{2}$ spaces.

In "Disturbance Rejection for Fractional-Order TimeDelay Systems" an equivalent-input-disturbance- (EID-) based disturbance rejection method for fractional-order time-delay systems is presented. First, a modified state observer is applied to reconstruct the state of the fractionalorder time-delay plant. Then, a disturbance estimator is designed to actively compensate for the disturbances. By constructing a novel monochromatic Lyapunov function and using direct Lyapunov approach, the stability analysis and controller design algorithm are derived in terms of LMI technique.

In "Dynamic Hedging Based on Fractional Order Stochastic Model with Memory Effect" the authors construct the minimum variance model for the estimation of the optimal hedge ratio based on the stochastic differential equation. At the same time, also by considering memory effects, the continuous-time hedge model with memory based on the fractional order stochastic differential equation driven by a fractional Brownian motion to estimate the optimal dynamic hedge ratio was established.

In "An Operational Matrix of Fractional Differentiation of the Second Kind of Chebyshev Polynomials for Solving Multiterm Variable Order Fractional Differential Equation" a method to solve multiterm variable order fractional differential equation based on the second kind of Chebyshev polynomials has been proposed. The main idea of this method is to derive a kind of operational matrices of variable order 
fractional derivative for the second kind of Chebyshev polynomials.

In "Fractional-Order Two-Port Networks" the concept of fractional-order two-port networks with particular focus on impedance and admittance parameters, showing how to transform a $2 \times 2$ impedance matrix with fractional-order impedance elements into an equivalent matrix with all elements represented by integer order impedances, has been introduced.

"Synchronization for a Class of Uncertain Fractional Order Chaotic Systems with Unknown Parameters Using a Robust Adaptive Sliding Mode Controller" deals with the synchronization of a class of fractional order chaotic system with unknown parameters and external disturbance. Based on the Lyapunov stability theory, a fractional order sliding mode is constructed and a controller is proposed to realize chaos synchronization.

In "Nonlocal Transport Processes and the Fractional Cattaneo-Vernotte Equation" an alternative representation of the Cattaneo-Vernotte equation using the fractional calculus approach, where the spatial-time derivatives of fractional order are approximated using the Caputo 1 type derivative in the range $(0,2]$, is presented.

"Investigation Progresses and Applications of Fractional Derivative Model in Geotechnical Engineering" is an overview aimed first at model structure and parameter determination in combination with application cases based on fractional calculus was provided. Furthermore, the review paper shed light on the practical application aspects of deformation analysis of circular tunnel, rheological settlement of subgrade, and relevant loess researches subjected to the achievements acquired in geotechnical engineering.

"On the General Solution of Impulsive Systems with Hadamard Fractional Derivatives" is concerned with the solution for impulsive differential equations with Hadamard fractional derivatives. The general solution of this impulsive fractional system is found by considering the limit case in which impulses approach zero.

In "Construction of Fractional Power Series Solutions to Fractional Boussinesq Equations Using Residual Power Series Method" the authors generalize the idea of RPSM to solve any-order time-space fractional differential equations in high dimensional space with initial value problems in $R n$. Successively by using RPSM, they obtain FPS solutions of fourth-, sixth-, and $2 n$ th-order time-space fractional Boussinesq equations in $R$ and fourth-order time-space fractional Boussinesq equations in $R^{2}$ and $R^{n}$.

In "Composition Formulas of Bessel-Struve Kernel Function" the objective is to study and develop the generalized fractional calculus operators involving Appell's function $F_{3}(\cdot)$ due to Marichev-Saigo-Maeda.

In "The General Solution of Impulsive Systems with Caputo-Hadamard Fractional Derivative of Order $q \epsilon$ $\mathbb{C}(\mathfrak{R}(q) \in(1,2))$ " motivated by some preliminary works about general solution of impulsive system with fractional derivative, the generalized impulsive differential equations with Caputo-Hadamard fractional derivative of $q$ are further studied by analyzing the limit case (as impulses approach zero).
In "Compact Wide Frequency Range Fractional-Order Models of Human Body Impedance against Contact Currents" three circuit models using constant phase elements are investigated to represent the human body impedance against contact currents from $40 \mathrm{~Hz}$ to $110 \mathrm{MHz}$. The parameters required to represent the impedance are determined using nonlinear least squares fitting (NLSF) applied to the averaged human body impedance datasets.

In "Study on Time-Dependent Behavior of Granite and the Creep Model Based on Fractional Derivative Approach Considering Temperature" considering the damage evolution process, a new creep constitutive model is proposed to describe the creep property of granite at different temperatures based on fractional derivative. The parameters of the new creep model are determined on the basis of the experimental results of the two granites.

Riccardo Caponetto Juan J. Trujillo

Josè A. Tenereiro Machado 


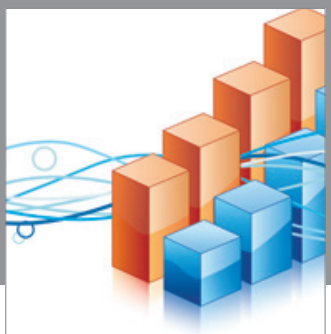

Advances in

Operations Research

vatem alat4

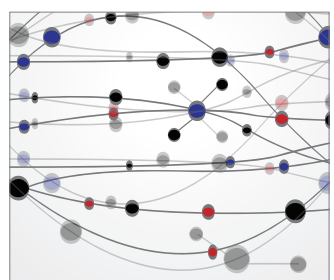

\section{The Scientific} World Journal
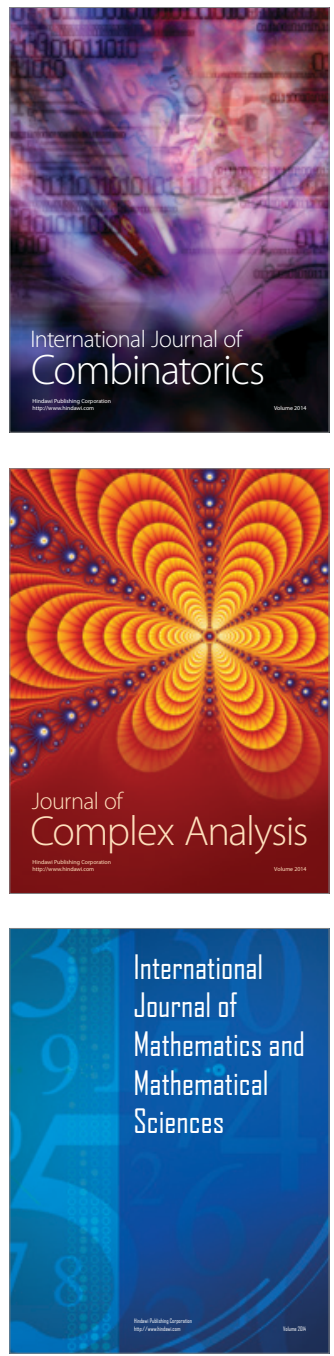
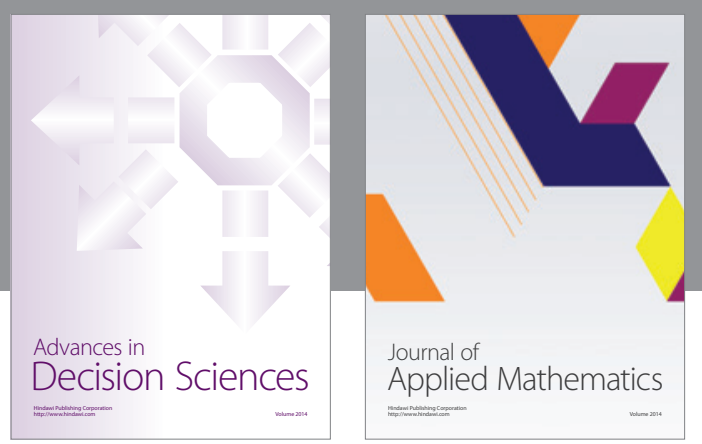

Algebra

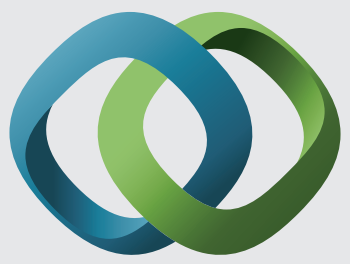

\section{Hindawi}

Submit your manuscripts at

http://www.hindawi.com
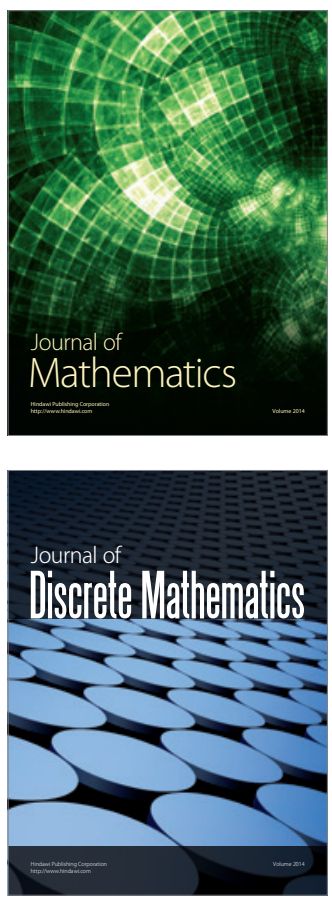

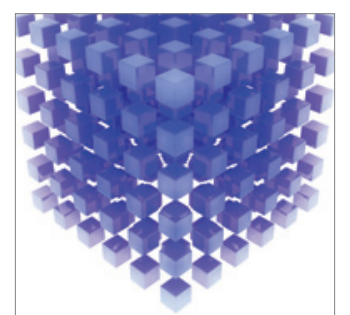

Mathematical Problems in Engineering
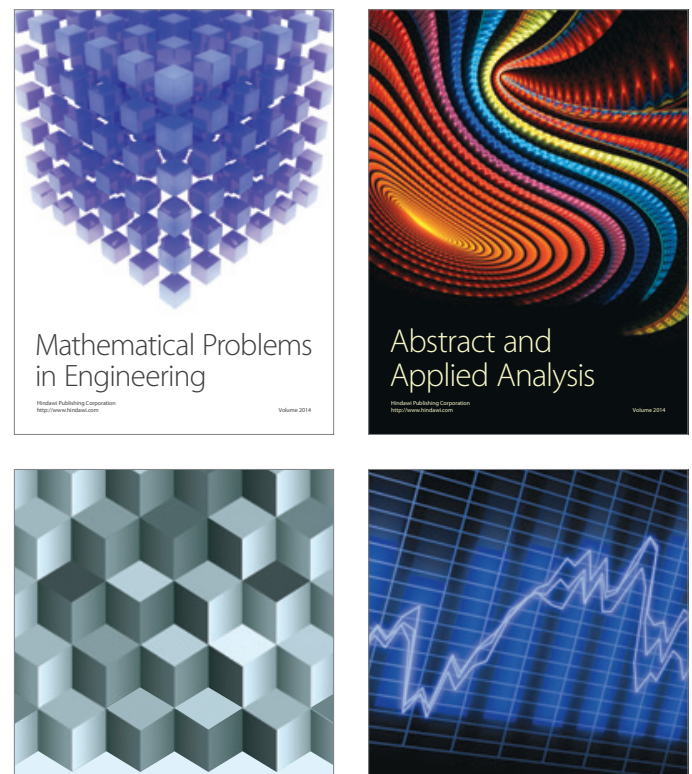

Journal of

Function Spaces

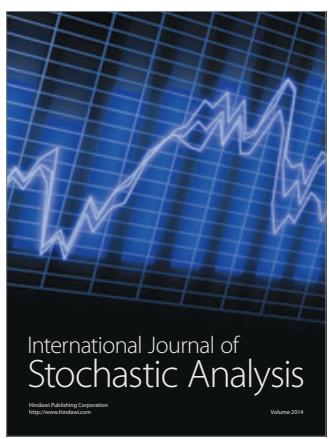

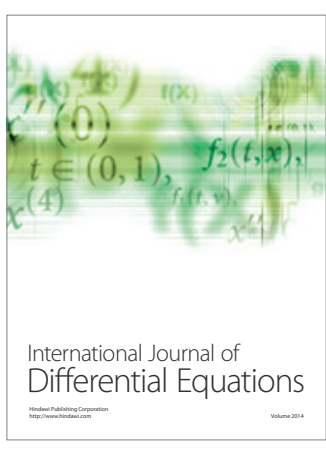
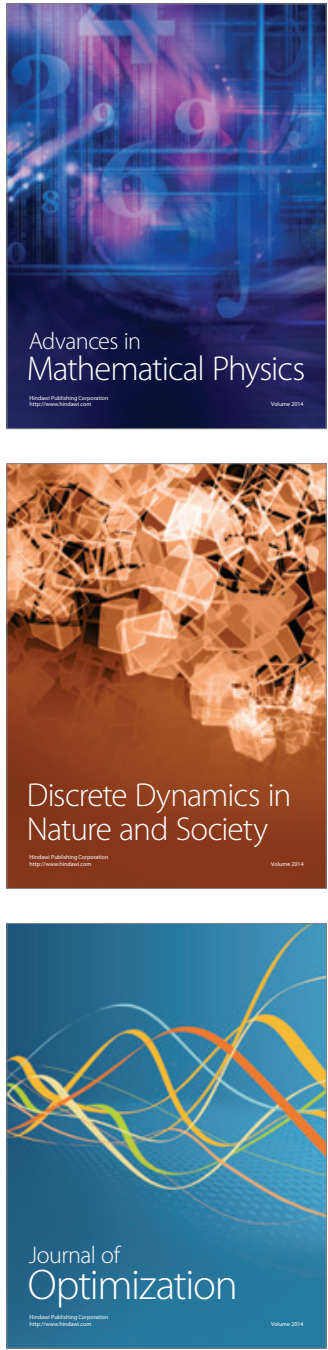